\title{
The Comparison of Entamoeba gingivalis Presence in Healthy and Periodontitis Patients by Using Direct Examination and PCR Methods
}

\author{
Mahmoud Rahdar (iD ${ }^{1,}$,, Salman Abolfazli-Karizi ${ }^{2}$ and Hootan Pedram ${ }^{3}$ \\ ${ }^{1}$ Infectious and Tropical Diseases Research Center, Health Research Institute, Ahvaz Jundishapur University of Medical Sciences, Ahvaz, Iran \\ ${ }^{2}$ Periodantics Department, Dentistary Faculty, Jundishapur University of Medical Sciences, Ahvaz, Iran \\ ${ }^{3}$ Dentistry Faculty, Jundishapur University of Medical Sciences, Ahvaz, Iran \\ "Corresponding author: Infectious and Tropical Diseases Research Center, Health Research Institute, Ahvaz Jundishapur University of Medical Sciences, Ahvaz, Iran. Email: \\ rahdar-m@ajums.ac.ir
}

Received 2018 November 04; Revised 2018 December 29; Accepted 2019 January 21.

\begin{abstract}
Background: The most frequent protozoan in the buccal cavity is Entamoeba gingivalis and more than 50\% of the world population are infected with it. The exact role of E. gingivalis in periodontal diseases remains uncertain.

Objectives: This cross-sectional descriptive study was conducted to compare the presence of E. gingivalis in healthy individuals and patients.

Methods: Twenty-five patients with periodontitis and 25 healthy controls were selected and their saliva and plaque samples were examined by microscopy and PCR methods.

Results: Only 3 out of the 25 patient samples were positive in microscopy examination, and four patient and two control group samples were positive in the PCR technique. There were no significant differences between the two groups $(\mathrm{P}>0.05)$ using Chisquare test.

Conclusions: : In the current study, E. gingivalis was not found to cause periodontal diseases. Studies with larger sample sizes are required to define the exact pathogenic role of E. gingivalis.
\end{abstract}

Keywords: Amoebiasis, Periodontitis, Oral Cavity, PCR

\section{Background}

Many people suffer from periodontitis and other dental diseases. Periodontitis is identified by gum inflammation, halitosis, pain, bleeding and loss of teeth (1). It is estimated that $5 \%-20 \%$ of the world population suffer from at least one oral disease (2). Several factors have been identified as the pathogenic agents for diseases including viruses, bacteria, fungi and parasites. The possible role of oral protozoa has been investigated yielding controversial results (1). Two common oral cavity protozoa are Entamoeba gingivalis and Trichomonas tenax, whose role in causing oral and dental diseases remains controversial.

E. gingivalis was first isolated and described by Gros (3). Many investigations reported E. gingivalis in 30\% - 80\% of patients with the periodontal diseases $(1,4)$, while it was undetectable in healthy controls $(4,5)$. E. gingivalis infection was reported in oropharynx as the commensal scavenger organism and in patients with poor oral sanitation, immunocompromised patients (6) and diabetic patients (7). The infection is acquired by the transmission of the trophozoite via oral contact (8). The parasite is also found commensal in calculus plaque and saliva (9). There is controversy regarding the pathogenesis of this parasite because it has been found in healthy individuals as well (4). The existence of E. gingivalis in periodontal disorder patients is more than healthy individuals and it may be suggested that it plays an important role in periodontal diseases as a pathogen or opportunistic agent (10).

By using direct examination and culture, previous studies showed that the prevalence of E. gingivalis in patients was only $1 \%$ (11).

\section{Objectives}

However, the goal of this study was to compare the presence of E. gingivalis in periodontal patients and healthy individuals.

\section{Methods}

\subsection{Patients}

In this descriptive case-control study, 25 patients including 17 (68\%) women and 8 (32\%) men with the mean age 
of 49.16 years and moderate to severe periodontitis were chosen. The grade of periodontitis severity was confirmed by a dental specialist.

The inclusion criteria included (1) having more than 20 teeth, (2) having periodontitis injury with deep pocket (more than $3 \mathrm{~mm}$ ) and (3) having attachment loss more than $2 \mathrm{~mm}$. The exclusion criteria comprised consuming systemic antibiotics in the past two months, having diabetes, using immunosuppressive drugs, smoking, being pregnant and having systemic, heart or respiratory diseases.

Twenty-five healthy volunteers were also chosen as the control group. The control group was matched with the patient group in terms of age and sex. Dental curettage and saliva (sputum) were collected in ethanol from patients and transferred to the parasitology department for trichrome staining and molecular experiments.

The patients' condition for participation in the study was evaluated by a dental specialist. The consent form for participation was filled out by the patients and the control group. The ethical code number was U-93048.

\subsection{Polymerase Chain Reaction (PCR)}

DNA extraction was performed in all the 50 samples ( 25 patients and 25 control) by Qiagen kits according to the manufacturer's instructions. The primers used for PCR included forward $5^{\prime}$-GAATAGGCGCGCATTTCGAACAGG- ${ }^{\prime}$ and reverse $5^{\prime}$-TCCCACTAGTAAGGTACTACTC-3. The primers amplified a fragment of 18S-SSU rDNA. The volume of each reaction was $25 \mu \mathrm{L}$ consisted of $5 \mu$ DNA sample, $3 \mu$ of the two primers (each one $1.5 \mu), 10 \mu$ premix $\left(\mathrm{MgCl}_{2}\right.$, dNTP, Tag enzyme, Tris) and $7 \mu$ distilled water. The thermocycler program was $7.5 \mathrm{~min}$ at $95^{\circ} \mathrm{C}$ for primary denaturation followed by 40 cycles at $95^{\circ} \mathrm{C}$ for $1 \mathrm{~min}, 30 \mathrm{~s}$ at $55^{\circ} \mathrm{C}$ for annealing, $30 \mathrm{~s}$ at $72^{\circ} \mathrm{C}$ for extension and $5 \mathrm{~min}$ at $72^{\circ} \mathrm{C}$ for final extension. The PCR product was run on $1.5 \%$ gel and the bands were visualized by gel documentation system.

\subsection{Statistical Analysis}

$T$-test was used for statistical analysis, and P value less than 0.05 was considered significant.

\section{Results}

Seventeen women and eight men were included in the patient group with the mean age of 49.16 years. In microscopic examination, 3 out of 25 (12\%) patient samples were positive for E. gingivalis, but in the control group samples no positive cases were found (Table 1 ).

\begin{tabular}{|c|c|c|c|c|c|}
\hline \multirow{2}{*}{ Variables } & \multirow{2}{*}{$\mathbf{N}$} & \multicolumn{2}{|c|}{ Patients } & \multicolumn{2}{|c|}{ Controls } \\
\hline & & Positive & Negative & Positive & Negative \\
\hline Men & 8 & $1(12.5)$ & $7(87.5)$ & 0 & $8(100)$ \\
\hline Women & 17 & $2(11.7)$ & $15(88.2)$ & 0 & $17(100)$ \\
\hline Total & 25 & $3(12)$ & $22(88)$ & 0 & $25(100)$ \\
\hline
\end{tabular}

${ }^{\mathrm{a}}$ Values are expressed as No. (\%).

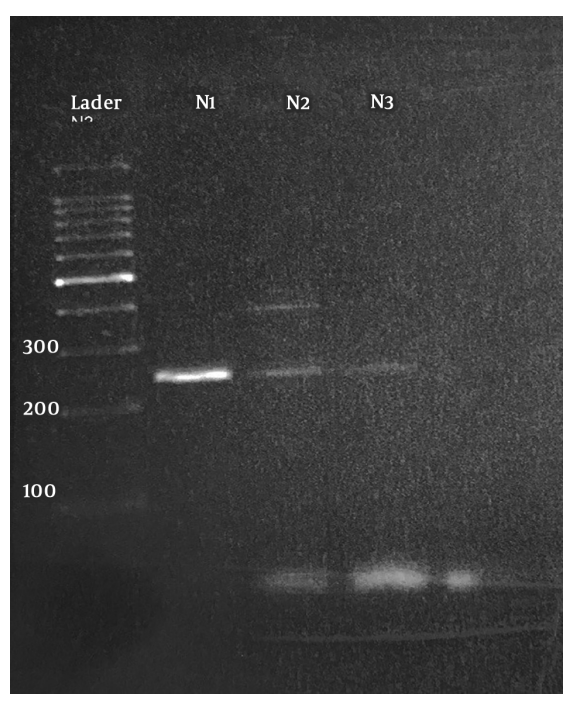

Figure 1. The 250-bp band of E. gingivalis N1-N3 positive samples

\subsection{PCR Findings}

Amplification of 18S-SSU rDNA gene revealed that 4 out of 25 (16\%) patient samples showed a 250-bp fraction pertaining to E. gingivalis (Figure 1). Also, 2 (8\%) samples of the control group showed a 250-bp fragment in PCR analysis. The prevalence rates of E. gingivalis were $16 \%$ and $8 \%$ in the patients and the controls, respectively. Statistical analysis of the data showed no significant difference between the patients and controls and between the two genders ( $P>0.05)$. The related risk (RR) was 2.19 , and 95\% lower and upper confidence intervals were 0.36 and 13.21 , respectively. All the positive samples in the direct examination were also positive in PCR and showed 250-bp band in gel electrophoresis.

\section{Discussion}

Periodontitis is one of the important dental diseases in many parts of the world. The prevalence of the disease increases by age and it is estimated that $50 \%$ of people above 30 years of age are involved with this condition (12). Many 
pathogenic agents as well as systemic and immunosuppressive diseases can cause major periodontal problems (13). Among the current protozoa of the oral cavity, E. gingivalis is the most frequent parasite. The role of this parasite in inducing periodontal diseases is not exactly clear and remains controversial. To define the pathogenic role of the parasite, appropriate treatment schedules should be set up by dentists.

We found no significant difference between the two examined groups $(\mathrm{P}>0.05)$, that is, the presence of E. gingivalis cannot induce periodontal lesions and other factors are involved. Wantland et al. reported that $56 \%$ of patients with periodontal diseases had E. gingivalis in their saliva and plaque by culture (14). He indicated the ability of E. gingivalis to cytolyze and ingest epithelial cell fragment of nuclear and red and white blood cells (14). In our study, $16 \%$ of the patients were infected with E. gingivalis and our results are different from those of Wantland et al. study because of different sample sizes. Wantland et al. selected 300 samples from healthy individuals and patients, but in current study, we collected 50 samples. Wantland et al. used different age groups for his study ranging from 6 to 80 years old.

Studies showed that the presence of parasites raises with increasing age. In the current study, the age of patients ranged from 20 to 50 years old. The method of diagnosis also was different. Bass and Johns indicated that E. gingivalis can be considered as a pathogen in the buccal cavity. They showed the existence of the amoeba in pyorrhea alveolaris patients but not in healthy volunteers (15). Linke et al. found that E. gingivalis serves an important role in periodontal diseases (16). These findings are not in line with our results. Maraghi study reported the main causative agent of periodontitis is Candida albicans (11). On the other hand, there are some articles indicating that $E$. gingivalis is a saprophyte protozoan and it can be detected in many healthy individuals without any periodontal disorders.

The results of this study showed no significant difference between the two groups in terms of the presence of the protozoan. It seems that E. gingivalis is not the pathogenic agent for gingivitis. Also, there was not any difference between men and women in E. gingivalis infection. Some studies reported a direct relationship between the severity of periodontal disease and presence of E. gingivalis in saliva and dental plaques $(9,17-19)$. It is suggested that Entamoeba gingivalis can produce dental biofilms to develop periodontitis (20). On the other hand, MielnikBłaszczak indicated that there was no significant correlation between dental caries and the presence of E. gingivalis (21). The presence of the amoeba in the buccal cavity in three groups including healthy periodontium, orthodontic treatment group and patients with periodontal diseases was studied. The prevalence of infection in these groups was $54.3 \%, 81.2 \%$ and $73.5 \%$, respectively, showing no significant difference between the groups (22).

There is a hypothesis that E. gingivalis may amplify in periodontitis lesions more than in healthy periodontium (23). Also, Dao et al. presented no significant differences between a healthy control group and patients with regards to E. gingivalis presence in the buccal cavity. They collected plaque scraping samples from 113 periodontal patients and 96 healthy controls. The rates of E. gingivalis infection in patients and controls were 59\% and 32\%, respectively. They showed no significant difference between the two groups (24). This finding is in agreement with our study results. The presence of E. gingivalis in the oral cavity of healthy individuals has called into question E. gingivalis pathogenesis. Further studies are necessary to determine the precise role of E. gingivalis as the causative agent of periodontitis.

\section{Acknowledgments}

This article is extracted from the Medical Dentistry thesis of Hootan Pedram (Dentistry Medical student) as approval number code U-93048 and is supported financially by Ahvaz Jundishapur University of Medical Sciences.

\section{Footnotes}

Authors' Contribution: Mahmoud Rahdar: Designing, PCR and writing. Salman Abolfazli-Karizi: Diagnosis of patients. Hootan Pedram: Sample collection and PCR.

Conflict of Interests: There is not any conflict of interest. Ethical Considerations: The agreement form for participation was filled by patients and the control group. The ethical code number is U-93048.

Financial Disclosure: This article is extracted from the medical dentistry thesis of Hootan Pedram (dentistry medical student) as approval number code U-93048 and is supported financially by Ahvaz Jundishapur University of Medical Sciences.

Funding/Support: This project is supported financially by Ahvaz Jundishapur University of Medical Sciences.

\section{References}

1. Bonner M, Amard V, Bar-Pinatel C, Charpentier F, Chatard JM, Desmuyck Y, et al. Detection of the amoeba Entamoeba gingivalis in periodontal pockets. Parasite. 2014;21:30. doi: 10.1051/parasite/2014029. [PubMed: 24983705]. [PubMed Central: PMC4077299].

2. Burt B; Research; Therapy Committee of the American Academy of. Position paper: Epidemiology of periodontal diseases. J Periodontol. 2005;76(8):1406-19. doi: 10.1902/jop.2005.76.8.1406. [PubMed: 16101377]. 
3. Gros G. Fragments d'helminthologie et de physiologie microscopique. Bull Soc Imp Nat Moscou. 1849;22:549-573.

4. Trim RD, Skinner MA, Farone MB, Dubois JD, Newsome AL. Use of PCR to detect Entamoeba gingivalis in diseased gingival pockets and demonstrate its absence in healthy gingival sites. Parasitol Res. 2011;109(3):857-64. doi: 10.1007/s00436-011-2312-9. [PubMed: 21400116].

5. Lucht E, Evengard B, Skott J, Pehrson P, Nord CE. Entamoeba gingivalis in human immunodeficiency virus type 1-infected patients with periodontal disease. Clin Infect Dis. 1998;27(3):471-3. doi: 10.1086/514709. [PubMed: 9770142].

6. Bhaijee F, Bell D. Entamoeba gingivalis in acute osteomyelitis of the mandible. Case Rep Med. 2011;2011:357301. doi: 10.1155/2011/357301. [PubMed: 21785604]. [PubMed Central: PMC3139901].

7. Chomicz L, Szubinska D, Piekarczyk J, Wojtowicz A, Piekarczyk B, Starosciak B, et al. [Occurrence of oral subclinical infections in insulin treated diabetics]. Wiad Parazytol.2004;50(2):177-80. Polish. [PubMed: 16859022].

8. Ghabanchi J, Zibaei M, Afkar MD, Sarbazie AH. Prevalence of oral Entamoeba gingivalis and Trichomonas tenax in patients with periodontal disease and healthy population in Shiraz, Southern Iran. Indian J Dent Res. 2010;21(1):89-91. doi: 10.4103/0970-9290.62821. [PubMed: 20427914].

9. Gharavi MJ, Hekmat S, Ebrahimi A, Jahani MR. Buccal cavity protozoa in patients referred to the faculty of dentistry in Tehran, Iran. Iran J Parasitol. 2006;1(1):43-6.

10. Chen JF, Wen WR, Liu GY, Chen WL, Lin LG, Hong HY. Studies on periodontal disease caused by Entamoeba gingivalis and its pathogenetic mechanism. Rev China Med J. 2001;114(12):12-5.

11. Rahdar M, Maraghi S, Azizi A, Mousanejad F, Vazirianzadeh B. [A study on the frequency of buccal cavity protozoa in patients with periodontitis and gingivitis in Ahvaz, southwest of Iran in 2009].Jundishapur J Health Sci. 2013;4(4):85-90. Persian.

12. Eke PI, Dye BA, Wei L, Thornton-Evans GO, Genco RJ; Cdc Periodontal Disease Surveillance workgroup: James Beck. Prevalence of periodontitis in adults in the United States: 2009 and 2010. J Dent Res. 2012;91(10):914-20. doi: 10.1177/0022034512457373. [PubMed: 22935673].

13. Shangase SL, Mohangi GU, Hassam-Essa S, Wood NH. The association between periodontitis and systemic health: An overview. SADJ. 2013;68(1):8.10-2. [PubMed: 23951755].

14. Wantland WW, Wantland EM, Remo JW, Winquist DL. Studies on human mouth protozoa. J Dent Res. 1958;37(5):949-50. doi: 10.1177/00220345580370052601. [PubMed: 13587822].

15. Bass CC, Johns FM. Pyorrhea dentalis and alveolaris: Specific cause and treatment. $J$ Am Med Assoc. 1915;64(7):553-8. doi: 10.1001/jama.1915.02570330001001

16. Linke HA, Gannon JT, Obin JN. Clinical survey of Entamoeba gingivalis by multiple sampling in patients with advanced periodontal disease. Int J Parasitol.1989;19(7):803-8. doi:10.1016/0020-7519(89)900696. [PubMed: 2592147].

17. Huang W, Shi JL, Li CL, Chen B, Shao L], Chen L, et al. [Entamoeba gingivalis infection among college students in Tangshan]. Zhongguo Ji Sheng Chong Xue Yu Ji Sheng Chong Bing Za Zhi. 2009;27(1):51-3. 61. Chinese. [PubMed: 19459501].

18. Ibrahim S, Abbas R. Evaluation of Entamoeba gingivalis and Trichomonas tenax in patients with periodontitis and gingivitis and its correlation with some risk factors. J Baghdad Coll Dentistry. 2012;24(3):158-62.

19. Chen JF, Liu GY, Wen WR, Chen C. [Studies on the continuous culture and pathogenicity of Entamoeba gingivalis]. Zhongguo Ji Sheng Chong Xue Yu Ji Sheng Chong Bing Za Zhi. 2000;18(2):84-6. Chinese. [PubMed: 12567722].

20. De Albuquerque Junior RLC, Cláudia M, De Santana WA, Silva FA. Incidence of Entamoeba gingivalis and Trichomonas tenax in samples of dental biofilm and saliva from patients with periodontal disease. RGO-Rev Gaúcha Odontol, Porto Alegre. 2011;59(1)

21. Mielnik-Błaszczak M, Rzymowska J, Michałowski A, SkawińskaBednarczyk A, Błaszczak J. Entamoeba gingivalis-prevalence and correlation with dental caries in children from rural and urban regions of Lublin Province, Eastern Poland. Ann Agr Env Med. 2018;25(4):656-8. doi: 10.26444/aaem/80403. [PubMed: 30586973].

22. Garcia G, Ramos F, Maldonado J, Fernandez A, Yanez J, Hernandez L, et al. Prevalence of two Entamoeba gingivalis ST1 and ST2-kamaktli subtypes in the human oral cavity under various conditions. Parasitol Res. 2018;117(9):2941-8. doi: 10.1007/s00436-018-5990-8. [PubMed: 29987412].

23. Gottlieb DS, Miller LH. Entamoeba gingivalis in periodontal disease. J Periodontol. 1971;42(7):412-5. doi: 10.1902/jop.1971.42.7.412. [PubMed: 5283012].

24. Dao AH, Robinson DP, Wong SW. Frequency of Entamoeba gingivalis in human gingival scrapings. Am J Clin Pathol. 1983;80(3):380-3. doi: 10.1093/ajcp/80.3.380. [PubMed: 6881102]. 